Annales Geophysicae (2001) 19: 1007-1017 (C) European Geophysical Society 2001

\title{
Mesospheric turbulent velocity estimation using the Buckland Park MF radar
}

\author{
D. A. Holdsworth ${ }^{1}$, R. A. Vincent ${ }^{2}$, and I. M. Reid ${ }^{2}$ \\ ${ }^{1}$ Atmospheric Radar Systems, 1/26 Stirling St, Thebarton, Australia \\ ${ }^{2}$ Dept. of Physics and Mathematical Physics, Adelaide University, Adelaide, Australia
}

Received: 11 October 2000 - Revised: 16 March 2001 - Accepted: 4 April 2001

\begin{abstract}
This paper investigates turbulent velocity estimation using the full correlation analysis (FCA) of spaced antenna (SA) data, and its application to the routine FCA observations of the Buckland Park MF (BPMF) radar. The effects of transmitter beamwidths are investigated, confirming the suggestions of previous authors that wide transmit beam widths lead to an overestimation of the turbulent velocity. The annual variation of the turbulent velocity is investigated, revealing an increase in turbulent velocity with height, and equinoctal minima and solstice maxima observed below $80 \mathrm{~km}$. Investigations of the turbulent velocities about the March diurnal tide maximum reveals a diurnal variation in phase with the zonal velocity. Harmonic analysis reveals this relationship exists between February and September. Descending power layers are also observed during this period. A number of mechanisms are proposed to describe these observations.
\end{abstract}

Key words. Meteorology and atmospheric dynamics (middle atmosphere dynamics; instruments and techniques) - Radio science (instruments and techniques)

\section{Introduction}

There exists a number of techniques for estimating turbulent random velocities and/or turbulent energy dissipation rates. These techniques include in-situ rocket measured fluctuations (e.g. Lübken et al., 1993; Lübken, 1997), measurements of the evolution of rocket released luminescents (e.g. Rees et al., 1972) and foil and chaff clouds (e.g. Wu et al., 1989), and radar based measurements. The radar techniques can be separated into those using measurements of the absolute signal power determined using calibrated radars (e.g. Van Zandt et al., 1978), those using measurements of line-of-sight velocity differences from meteor (e.g. Roper, 1966) and imaging Doppler interferometry scattering posi-

Correspondence to: D. A. Holdsworth

(dholdswo@atrad.com.au) tions (e.g. Roper and Brosnahan, 1997), and those using measurements based on the spectral width, such as from Doppler beam steering analysis (e.g. Hocking et al., 1983) and from spaced antenna (SA) full correlation analysis (e.g. Briggs, 1980; Manson et al., 1981; Hall et al., 1998).

Estimates of the turbulent velocity obtained from MF FCA observations are influenced by a number of effects that may result in a substantial overestimation of the actual turbulent velocity (e.g. Briggs, 1980; Vandepeer and Hocking, 1993). These effects result from the wide transmit beam widths used for MF radars, whose half-power half-widths are typically of the order of $20^{\circ}$ to $40^{\circ}$. The Buckland Park MF (BPMF) radar can be operated using transmit beam half-power halfwidth as low as $4.5^{\circ}$, which may allow the BPMF radar to reduce some of the effects producing overestimated turbulent velocities.

This paper describes the estimation of turbulent velocities using the BPMF radar. This study is motivated by renewed interest in estimating turbulent velocities from MF radars using FCA (e.g. Hall et al., 1998) and imaging Doppler interferometry (IDI) (e.g. Roper and Brosnahan, 1997), and from VHF radars using alternative correlation analyses to the FCA (e.g. Chau et al., 2000). Section 2 describes the FCA, its use in estimating turbulent velocities, and potential biases in the technique. Section 3 describes the BPMF radar, the routine analysis scheme, and the resulting annual and diurnal variation of the FCA turbulent velocity. Section 4 investigates possible mechanisms for the observed results. The relationship between turbulent velocities and turbulent energy dissipation rates (e.g. Hocking, 1996) is not addressed.

\section{Turbulent velocity estimation}

\subsection{The full correlation analysis (FCA)}

The full correlation analysis (FCA) has been employed for atmospheric wind velocity estimation using spaced antenna (SA) radars for over forty years (e.g. Briggs et al., 1950; 
Phillips and Spencer, 1955). The technique is currently applied using partial reflection returns from SA radars operating at VHF in the lower atmosphere $(2-20 \mathrm{~km})$ (e.g. Vincent et al., 1987) and MF/HF in the upper mesosphere and lower thermosphere (60-100 km) (e.g. Vincent and Lesicar, 1991). The technique assumes that contours of equal spatiotemporal correlation of the ground diffraction pattern resulting from the backscatter of a transmitted signal by atmospheric refractive index irregularities can be approximated by a family of ellipsoids (e.g. Briggs, 1984). The pattern is generally sampled at three non-colinear positions, and the temporal auto- and cross-correlation functions calculated using the complex signals recorded at these antennas are used to parameterize the spatio-temporal correlation function. The FCA produces two velocity estimates, namely the "apparent" and "true" velocities. The apparent velocity is calculated using the temporal lags to maximum cross correlation, and is susceptible to the effects of random changes and anisometry in the ground diffraction pattern. The true velocity allows for these effects and is, therefore, considered the better estimate of the actual velocity.

The FCA also parameterizes the spatial and temporal properties of the ground diffraction pattern. The temporal properties are described by the ground diffraction pattern lifetime in the reference frame of the pattern (the "pattern lifetime", $T_{0.5}$ ). The pattern lifetime provides the means for estimating turbulent velocities, as described in Sect. 2.2.

\subsection{Estimation of turbulent random velocities using the FCA}

Using a simplified model where the scattering irregularities exhibit an isotropic Gaussian distribution of random velocity components, as in the case of molecular velocities in gas, Briggs (1980) illustrated that the root-mean-square (RMS) random velocity component $v_{\text {rms }}$ is related to the pattern lifetime $T$ by

$\nu_{\mathrm{rms}}=\frac{\lambda \sqrt{2 \ln 2}}{4 \pi T}$

where $\lambda$ is the radar wavelength. Note that (1) is the correct expression for the use of complex receiver outputs. Briggs (1980) assumed amplitude only receiver outputs, and does not include the extra factor of $\sqrt{2}$ in the numerator. Alternatively, the Fourier transform relation between correlation functions and power-spectral widths (e.g. Bracewell, 1965) may be applied to relate $v_{\text {rms }}$ to the spectral half-power halfwidth $f_{0.5}$ in the frame of the diffraction pattern by

$$
v_{\mathrm{rms}}=\frac{\lambda f_{0.5}}{2 \sqrt{2 \ln 2}} \text {. }
$$

The spectral width provides the simplest tool for understanding the effects of various phenomena upon the estimate of $v_{\text {rms }}$, as in the absence of any complicating factors (such as those described in Sect. 2.3), it can be related directly to the distribution of radial velocities and the transmitter polar diagram.
Consider the situation where the scatterers exhibit a Gaussian distribution of random velocity components $\boldsymbol{V}^{\prime}=\left(u^{\prime}\right.$, $\left.v^{\prime}, w^{\prime}\right)$ about a mean component $\overline{\boldsymbol{V}}=(\bar{u}, \bar{v}, \bar{w})$. In the frame of the ground diffraction pattern, $\bar{u}=\bar{v}=\bar{w}=0$. If the scatterers are assumed isotropic and are observed by a radar employing a vertical beam with a transmitter half-power $e^{-1}$ width $\theta_{b}$, the $e^{-1}$ width of the resulting power spectrum in the frame of the ground diffraction pattern is given by

$f_{s} \approx 2 w^{\prime} \cos \theta_{b}+\max \left[u^{\prime}, v^{\prime}\right] \sin \theta_{b} / \lambda$

For narrow beam radars, (e.g. VHF radars $-\theta_{b} \approx 1-2^{\circ}$ ), $\cos \left(\theta_{b}\right) \approx 1$, and (3) reduces to

$f_{s} \approx 2 w^{\prime} / \lambda$.

Since, $f_{0.5}=f_{s} \sqrt{2 \ln 2}$, (4) is identical to (2) in replacing the isotropic turbulent velocity with the vertical component. It follows that the observed spectral width provides a good estimate of the vertical turbulent velocities for narrow beam radars with vertically directed beams when $\bar{u}=\bar{v}=\bar{w}=0$ (e.g. Hocking, 1983). However, for larger beam width radars (e.g. typical MF radars $-\theta_{b} \approx 20-40^{\circ}$ ), this estimate may contain significant contributions from the horizontal turbulent velocities.

In the more general case of a fixed-position finite-beamwidth radar observing an atmosphere with anisotropic turbulent random velocities and anisotropic scattering irregularities, (1) must be interpreted carefully. The major complicating factor in using (1) is that the radar does not observe the scatterers from the frame of the ground diffraction pattern. There is, therefore, a contribution to the spectral width due to the finite beam-width of the radar. This is known as "beam broadening" (e.g. Briggs, 1980; Hocking, 1983). Significant care is required to remove the effects of beam broadening from the estimate of $v_{\text {rms }}$ when using spectral widths (e.g. Hocking, 1983, 1988). Furthermore, such removal is complicated by scatterer aspect sensitivity which may reduce the effective beam-width of the combined transmitter and scatterer polar diagram, leading to an overestimation of the effected beam broadening. (e.g. Hocking, 1983, 1988). The FCA pattern lifetime $T_{0.5}$ provides an estimate of the correlation width in the frame of the ground diffraction pattern. This has the advantage that the effects of beam broadening are removed.

Substituting the FCA pattern lifetime into (1) yields

$v_{\mathrm{fca}}=\frac{\lambda \sqrt{2 \ln 2}}{4 \pi T_{0.5}}$,

which will hereafter be referred to as the FCA turbulent velocity. Although this equation has been derived using heuristic arguments based on the ground diffraction pattern, an identical formula has been derived using scattering theory (e.g. Holloway et al., 1997).

\subsection{Biases in FCA turbulent random velocity estimates}

The FCA estimate of the turbulent RMS velocity $v_{\text {fca }}$ is usually considered an overestimate. This is because (5) assumes 
a causal relationship between random changes in ground diffraction and the turbulent motions of the scattering irregularities. However, random changes in the ground diffraction pattern can be introduced by a number of effects, including:

1. variations in the horizontal or vertical wind velocity with height (e.g. Briggs, 1980),

2. wind field inhomogeneities (e.g. Sürücü et al., 1995), such as those introduced by gravity waves with scales of the order of the diameter of the radar viewing volume,

3. the evolution of the returned signal amplitudes from individual refractive index irregularities (e.g. Holdsworth, 1995),

4. "triangle size effect" (TSE) biases (e.g. Meek, 1990; Holdsworth, 1999), and

5. short period gravity waves (e.g. Meek and Reid, 1984; Hocking, 1988; Vandepeer and Hocking, 1993), which can contribute in three ways (e.g. Vandepeer and Hocking, 1993):

(a) vertical fluctuating motion of the wave (e.g. Murphy et al., 1993),

(b) vertical velocity variations across the radar beam (e.g. Murphy et al., 1993),

(c) the horizontal fluctuating motion of the wave (e.g. Hocking, 1996).

The evolution of the returned signal amplitudes can occur due to turbulent mixing, producing irregularities whose scattering characteristics grow, evolve, and eventually decay. The effects of finite scatterer lifetimes upon $v_{\text {fca }}$ were investigated by Holdsworth (1995) using the radar backscatter model of Holdsworth and Reid (1995). The results suggested that scatterer lifetimes of the order of seconds could produce significant biases in $v_{\text {fca }}$ for MF observations. However, it is expected that the effects of scatterer lifetimes may be more significant in the boundary layer where scatterer lifetimes of the order of 1 second are expected (e.g. Doviak et al., 1995). Another source of returned signal amplitude evolution is due to changes in the amplitude of the returns from individual scatterers due to the spatial power variation of the transmitter polar diagram, which Holdsworth and Reid (1995) suggested was the reason for non-zero turbulent velocity estimates obtained in their study when using zero model turbulent velocity. This effect is expected to be more significant for narrow beamwidth systems.

The TSE denotes the increase in the magnitude of the FCA true velocity often observed with increasing antenna spacing up to a maximum given by the actual wind velocity. This effect also results in an increase in $T_{0.5}$ with increasing spacing, suggesting that smaller antenna spacings can result in an underestimation of $T_{0.5}$, resulting in an overestimation of $v_{\text {rms }}$.

Hocking (1988) has previously estimated turbulent velocities using a Doppler spectral width (hereafter referred to as
Table 1. Experimental parameters used for routine spaced antenna (SA). Analysis for the Buckland Park MF radar

\begin{tabular}{lcc}
\hline Parameter & Day value & Night value \\
\hline Height resolution & $2 \mathrm{~km}$ & $2 \mathrm{~km}$ \\
Number of heights & 25 & 15 \\
Start height, km & 50 & 70 \\
Polarization & linear & linear \\
PRF, Hz & 100 & 20 \\
Coherent integrations & 40 & 8 \\
Effective sampling time, s & 0.4 & 0.4 \\
Number of samples & 280 & 280 \\
Record length, s & 112 & 112 \\
\hline
\end{tabular}

DSW) technique applied to data collected with the BPMF radar using a wide transmit beam and a receive beam width of $4.5^{\circ}$. Rough estimates of the contribution of gravity waves to the observed spectral widths were found to be height dependent, ranging from $10 \%$ to $50 \%$ between 84 and $92 \mathrm{~km}$. These results led Hocking (1988) to suggest that wave motions (rather than turbulent motions) are the major contributor to the turbulent velocity estimates below $84 \mathrm{~km}$, and to seriously doubt the validity of turbulent velocity estimates obtained using (5) from typical wide-beam MF radar systems.

\section{Application of the FCA to BPMF results}

\subsection{The Buckland Park MF radar}

The Buckland Park medium frequency (BPMF) radar (e.g. Briggs et al., 1969) is located $35 \mathrm{~km}$ north of Adelaide $\left(34^{\circ} 38^{\prime} \mathrm{S}, 138^{\circ} 29^{\prime} \mathrm{E}\right)$. The radar operates at a frequency of $1.98 \mathrm{MHz}$. The main antenna array consists of 89 crossed half-wave dipoles arranged in a square grid with spacing 91.4 $\mathrm{m}$, forming a filled circle of diameter $914 \mathrm{~m}$. The radar has recently been overhauled (e.g. Reid et al., 1995), involving the complete replacement of the antenna array as well as the transmitting and receiving system. The main array can now also be used for transmission, enabling the radar to operate as a true Doppler radar (e.g. Vandepeer and Reid, 1995).

The BPMF radar has been used for routine spaced antenna observations using the FCA since May 1996 (e.g. Holdsworth and Reid, 2001). The relevant radar operating parameters are shown in Table 1. The observation periods for FCA observations are shown in Table 2. Near-continuous observations were made during these observation periods, with some radar downtime allowed for scheduled antenna maintenance and campaign experiments. Transmission was performed using the north-south aligned antennas, resulting in a linearly polarised transmitted signal.

It was envisaged that the improved BPMF radar and the more restrictive antenna selection currently employed should result in a considerable improvement in data quality with respect to previous BPMF observations. This indeed has 
Table 2. Observation periods for routine spaced antenna (SA). Analysis for the Buckland Park MF radar

\begin{tabular}{lccc}
\hline Observation period & $\begin{array}{c}\text { Number of } \\
\text { antennas }\end{array}$ & $\begin{array}{c}\text { Transmit half-power } \\
\text { half-width (deg) }\end{array}$ & $\begin{array}{c}\text { Peak } \\
\text { Power (kw) }\end{array}$ \\
\hline $7 / 5 / 1996$ to $16 / 7 / 1997$ & 30 & 10 & 25 \\
$16 / 7 / 1997$ to $4 / 1 / 1998$ & 60 & 5 & 75 \\
$23 / 3 / 1998$ to $23 / 4 / 1998$ & 30 & 10 & 25 \\
$24 / 3 / 1998$ to $29 / 6 / 1998$ & 60 & 5 & 50 \\
$2 / 3 / 1999$ to $31 / 7 / 1999$ & 75 & 4.5 & 87.5 \\
$1 / 8 / 1999$ to present & 60 & 5 & 50 \\
\hline
\end{tabular}

proven to be the case. Furthermore, previous BPMF radar observations were performed using a number of different data acquisition systems using 8-bit digitisation, including one receiving system whose receivers exhibited significant non-linearity approaching the digitisation limits and suspected receiver characteristic differences. The use of 8-bit digitisation can produce significant TSE biases at upper and lower ranges where the effects of receiver saturation and coarse digitisation can become significant (e.g. Holdsworth, 1999). Furthermore, receiver non-linearity and characteristic differences can cause further TSE biases (e.g. Holdsworth, 1999).

Although quantitative estimates of the contributions of the sources of potential overestimation, discussed in Sect. 2.3, have not been made, we believe the transmit pulse length, beam width and short record lengths (112 s) used inhibit the contributions due to factors 1, 2, and 5 described in Sect. 2.3. Since the record length is of the order of the scatterer lifetimes proposed by Roper and Brosnahan (1997), the contributions due to factor 3 should also be small. Evaluation of the BPMF receiving system and the selection of appropriate receiving antennas to reduce TSE biases (e.g. Holdsworth and Reid, 2001), suggests contributions due to 4 will be small, but significant. We expect the contributions of short period gravity waves to be similar to the BPMF radar DSW observations of Hocking (1988). Although these observations were made using different experimental configuration and analysis procedures to those used in the current study, the effective beam-widths are similar and the analysis procedures use similar rejection criteria. The DSW spike removal routine rejects frequency bins indicative of specular scatter, while the FCA uses slow fading and oscillatory correlation function criteria (e.g. Briggs, 1984) for the same purpose. The DSW goodness of fit criterion is used to reject non-Gaussian spectra which may be indicative of gravity waves (e.g. Murphy et al., 1993). Given the Fourier relationship between spectra and correlation functions, it is reasonable to expect that non-Gaussian spectra will produce non-Gaussian correlation functions, which may be rejected from the FCA by the oscillatory correlation function criteria.
3.2 Effect of transmitter beamwidth on BPMF turbulent velocities

Between the 4th and 18th of November 1996 the BPMF radar was operated together with a "wide-beam" MF radar system with characteristics similar to the majority of the MF radars currently operating throughout the world (e.g. Hocking, 1997), such as the Christmas Island MF radar (e.g. Vincent and Lesicar, 1991). The major differences for the widebeam system were:

1. transmission was performed using a single pair of crossed dipoles of the BPMF antenna array, while reception was performed using three pairs of crossed dipoles arranged in the BPMF antenna array in an approximately equilateral triangle with spacings $182.8,204.4$ and $204.4 \mathrm{~m}$,

2. a peak transmit power of $10 \mathrm{~kW}$ was used,

3. circular polarisation was used upon transmission and reception.

The transmitter half-power $e^{-1}$ width of this system is approximately $40^{\circ}$. The data acquisition parameters used were identical to those of the BPMF radar.

The BPMF and wide-beam radar were operated during alternate two minute periods. Although the two radar clocks were synchronised at the start of the experiment, time drifts between the clocks resulted in periods where the acquisition periods overlapped. This effect, coupled with the lower transmit power of the wide-beam system, limited the usable height range to between 80 and $100 \mathrm{~km}$, and therefore limited any in-depth investigation of the effects of beam-width upon the FCA.

Figure 1 shows a scatter plot of the half hourly average zonal and meridional velocities estimated using the BPMF and wide-beam systems. The results show good agreement, with correlations of 0.85 and 0.75 for the zonal and meridional components, respectively. The ratio of the BPMF to wide-beam velocities estimated using a total least squares fit are 1.01 and 1.05 for the zonal and meridional components, respectively, indicating the BPMF velocities are slightly larger than the wide-beam velocities. Although the antenna spacings used for both systems are comparable, the wider beam-width of the wide-beam system results in the widebeam observations having a smaller pattern scale than the BPMF observations. As a result, the antenna spacing for the wide-beam system is a better match to the average pattern scale than for the BPMF system, suggesting the wide-beam radar should be less susceptible to the TSE. Observations obtained using different antenna spacings with the BPMF system indicate that the BPMF radar antenna spacing used during this experiment can result in some velocity underestimation (e.g. Holdsworth and Reid, 2001). This suggests that there may be some underestimation of the wide-beam velocities, and hence, some overestimation of the wide-beam turbulent velocities. Figure 2 shows a scatter plot of the turbulent velocities estimated using the BPMF and wide-beam 

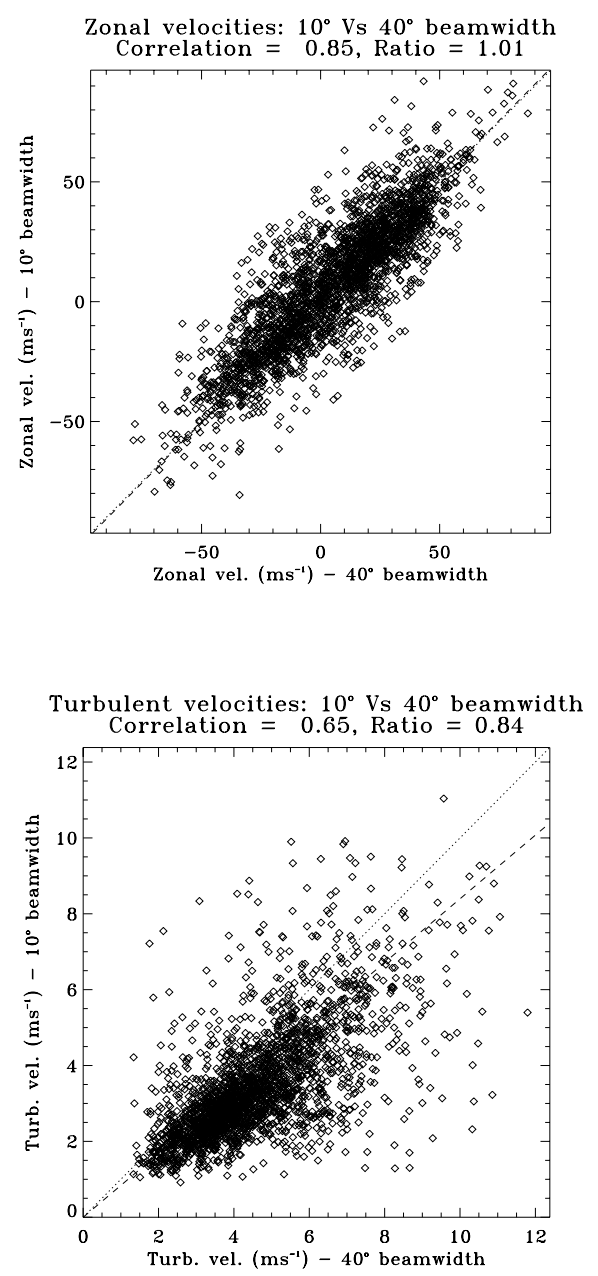
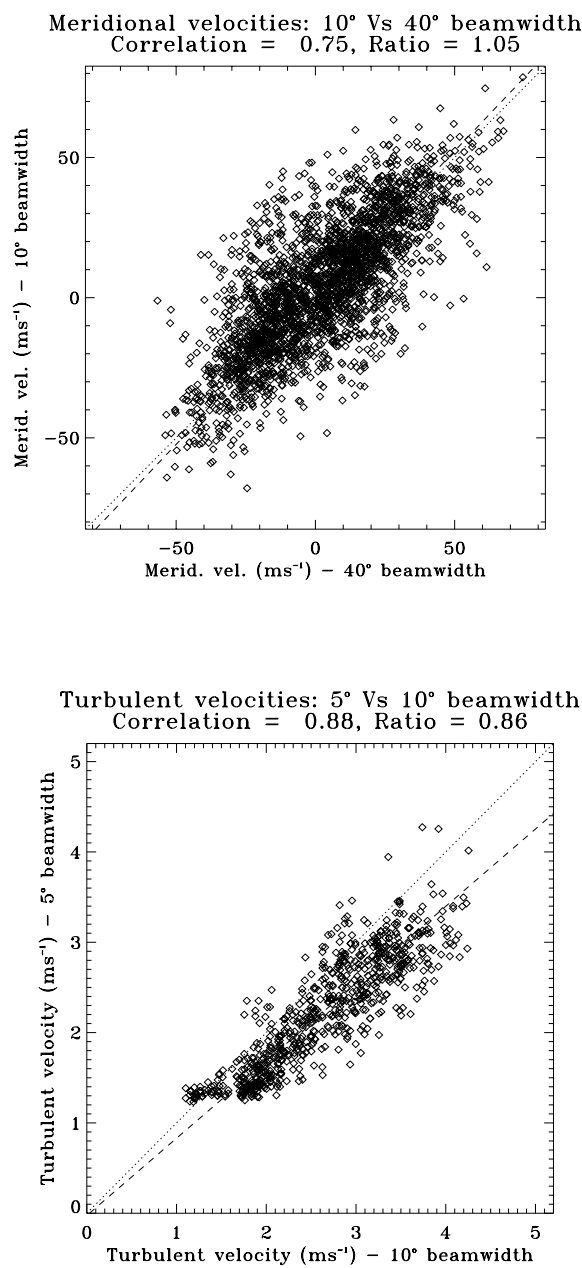

Fig. 1. Scatter plot of (left) zonal and (right) meridional velocities estimated using half hourly averaged estimates from 2-minute interleaved $10^{\circ}$ and $40^{\circ}$ observations during November 1996. The dotted line on both plots indicates $y=x$. The dashed line indicates the results of a total least squares fit, whose slope is indicated at the top of the plot.

Fig. 2. Scatter plot of turbulent velocities estimated using (left) half hourly averaged estimates from 2-minute interleaved $10^{\circ}$ and $40^{\circ}$ observations during November 1996, and (right) using $5^{\circ}$ and $10^{\circ}$ observations. Each data point for the bottom plot is obtained by comparing the turbulent velocity estimated using a $10^{\circ}$ beamwidth with that obtained in the same fortnightly period in a different year when the $5^{\circ}$ beamwidth was used. The dashed line indicates the results of a total least squares fit, whose slope is indicated at the top of the plot. systems. The ratio of the BPMF to wide-beam turbulent velocities estimated using a total least squares fit is 0.84 .

The use of different transmitter beamwidths throughout the period of the BPMF observations allows a further assessment of the effects of the beamwidth on the turbulent velocity estimate. Figure 2 shows scatter plots of turbulent velocities obtained using the beamwidths of $5^{\circ}$ and $10^{\circ}$. Each data point is obtained by comparing the turbulent velocity estimated using the $10^{\circ}$ beamwidth with that obtained in the same fortnightly period in a different year when the $5^{\circ}$ beamwidth was used. The fortnightly averages were formed by first averaging the data into half hourly averages, which were used to produce daily averages, which were, in turn, used to produce fortnightly averages. Application of a total least squares fit to these results produces a line with a 0.87 slope, suggesting the $10^{\circ}$ beamwidth estimates are $14 \%$ larger than the $5^{\circ}$ beamwidth estimates. It is important to stress that this analysis assumes that the year-to-year variations of the turbulent velocity is small, which may not necessarily be the case given observations of quasi-biennial oscillation (QBO) in the March diurnal tide parameters (e.g. Vincent et al., 1998). However, this assumption has been verified by performing the same analysis using fortnightly periods for different years when the same beamwidths were used. The results (not shown) suggest the year-to-year variability of the fortnightly averaged turbulent velocities are within $2 \%$.

\subsection{Seasonal behavior of BPMF turbulent velocities}

The fortnightly averaged zonal and turbulent velocities obtained using the routine BPMF FCA observations from March 1999 to June 2000 are shown in Fig. 3. The zonal velocities show eastward winter flows and northward summer flows, as previously observed by a number of authors at mid-latitude sites (e.g. Lesicar, 1993). The turbulent velocities increase with range. Solstice maxima are observed at about $80 \mathrm{~km}$, while equinoctal minima are observed below $80 \mathrm{~km}$. The turbulent velocities are in qualitative agreement with radar determined turbulent velocities and/or energy dissipation rates obtained at mid-latitude by other authors (e.g. Lesicar, 1993), as are the observed solstice maxima and equinoctal minima (e.g. Muller, 1968; Meek et al., 1985). The magnitude of the winter maxima is larger than the summer maxima, in contrast to the rocket observations of Lübken, 1997. The solstice maxima show a double maxima, which is also evident in the zonal velocities. The double 

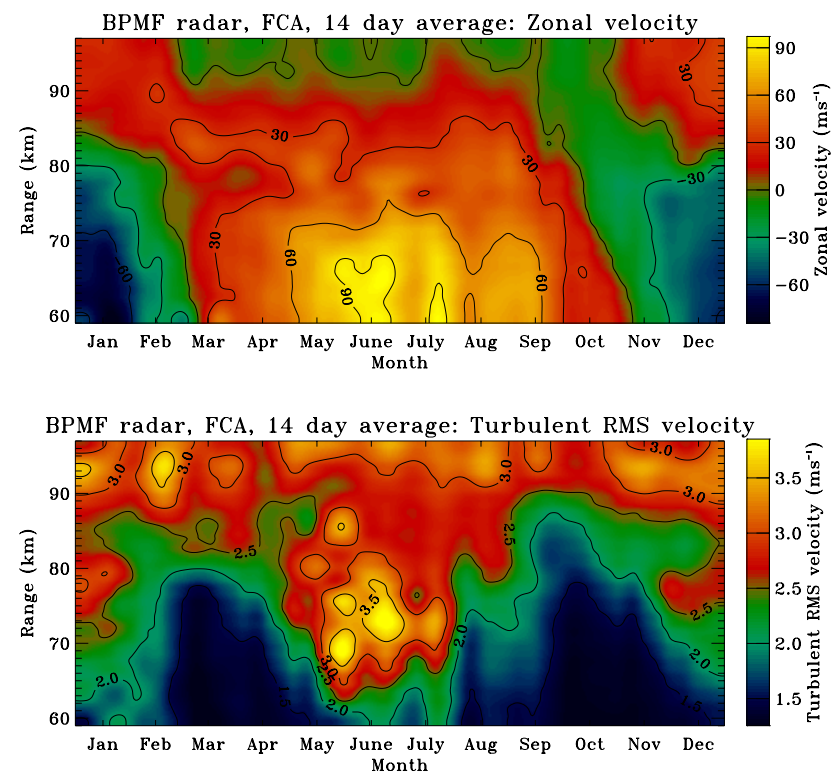

Fig. 3. Superposed epoch image plot of the annual variation of zonal (top) and turbulent (bottom) velocities between March 1999 and June 2000.

maxima are observed in both the turbulent and zonal velocities for each year of the current observations, with the zonal velocity maxima reaching up to $150 \mathrm{~m} \mathrm{~s}^{-1}$. The positions of the maxima change from year to year. These observations will be investigated in more detail in a later paper.

\subsection{Harmonic analysis of the BPMF FCA velocities}

The annual variation of the semidiurnal and diurnal tidal amplitudes and phases for Adelaide have previously been studied using harmonic analysis (e.g. Vincent et al., 1998). Harmonic analysis similar to that of Vincent et al. (1998) has been applied to the 1997 BPMF FCA data. This data set was chosen because it represents the only full year of observations and it is the most continuous data set as well. Half hourly averages of the zonal and meridional velocities have been used, with harmonic analysis performed using a 14day window shifted at 7-day intervals throughout the duration of the data set. Harmonic analysis was performed for each range gate where velocity estimates were available for at least $50 \%$ of the half hourly intervals used in the fitting procedure. No range averaging was used. Least squares fits of 12-, 24- and 48-hour harmonic components were then applied, with the zero phase corresponding to the maximum positive velocity excursion.

The amplitudes and phases of the diurnal zonal velocity for 1997 are shown in Fig. 4. These results show the same significant features as observed by Vincent et al. (1998), including amplitude maxima in March/April, July and October, a 4 hour phase increase from summer (January) to winter (July), a 4 hour phase decrease from summer to winter, and phase retardation around the winter solstice. The maximum amplitude observed during late March and April is
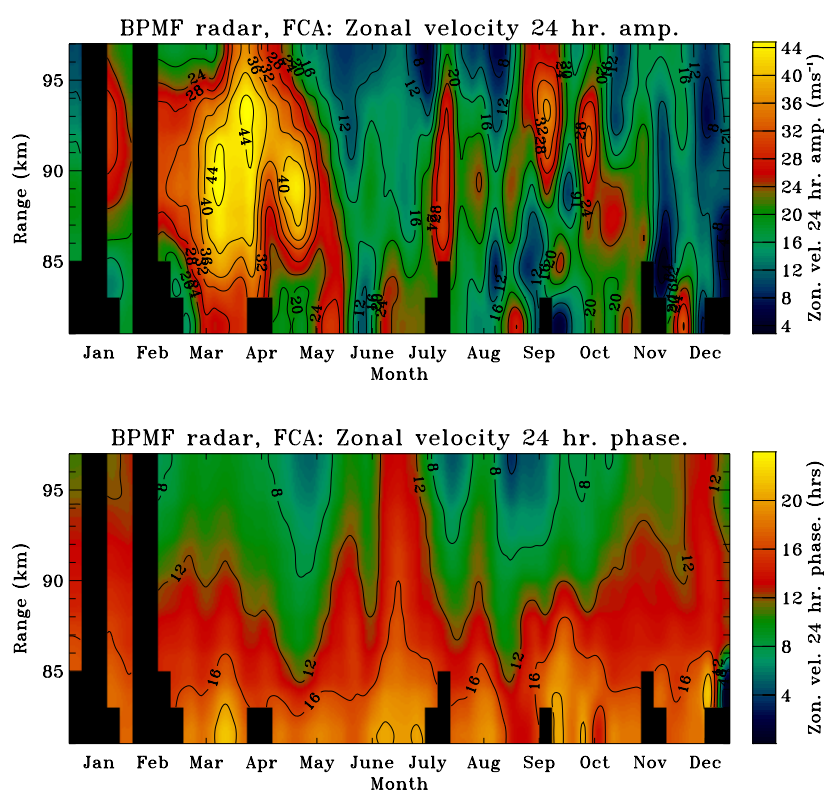

Fig. 4. Zonal velocity diurnal tide amplitude (top) and phase (bottom) estimated using harmonic analysis.

$\approx 45 \mathrm{~m} \mathrm{~s}^{-1}$. For reference, the maximum amplitudes observed from 1998 to 2000 are $\approx 30,60$, and $40 \mathrm{~m} \mathrm{~s}^{-1}$, suggesting evidence of the QBO observed in the diurnal tidal parameters by Vincent et al. (1998). These maximum amplitudes are larger than those observed by Vincent et al. (1998) using previous BPMF radar systems from 1984 to 1995. However, the Vincent et al. (1998) analysis used a 30-day fitting window with hourly velocity estimates, as opposed to the 14-day window with half hourly estimates used in the current study. The tidal amplitudes resulting from a larger fitting window usually result in smaller amplitudes. The current results support the hypothesis of Holdsworth and Reid (2001), that the FCA results presented in this paper are less susceptible to TSE introduced velocity underestimations than previous BPMF radar systems.

\subsection{Diurnal variation of BPMF turbulent velocities}

The half hourly BPMF FCA data for March 1999 have been superposed into a single "mean" day to reduce the effects of gravity and planetary wave motions. The resulting zonal and meridional velocities are displayed as an image plot in Fig. 5. The diurnal tide is clearly observed in both velocity components. The corresponding plot for the turbulent velocity $v_{\mathrm{fca}}$, estimated using (5), is shown in Fig. 6. This plot reveals a clear diurnal variation in $v_{\mathrm{fca}}$, with the phase of maximum $v_{\text {fca }}$ exhibiting good agreement with the phase of maximum zonal velocity. Although this behavior is also observed in the 1997, 1998 and 2000 data sets, the 1999 data depicts this behavior most clearly, presumably due to the fact that the odd numbered years show larger diurnal tidal maxima (in association with the QBO), and the narrower transmitter beamwidth used in 1999 in comparison to 1997. 

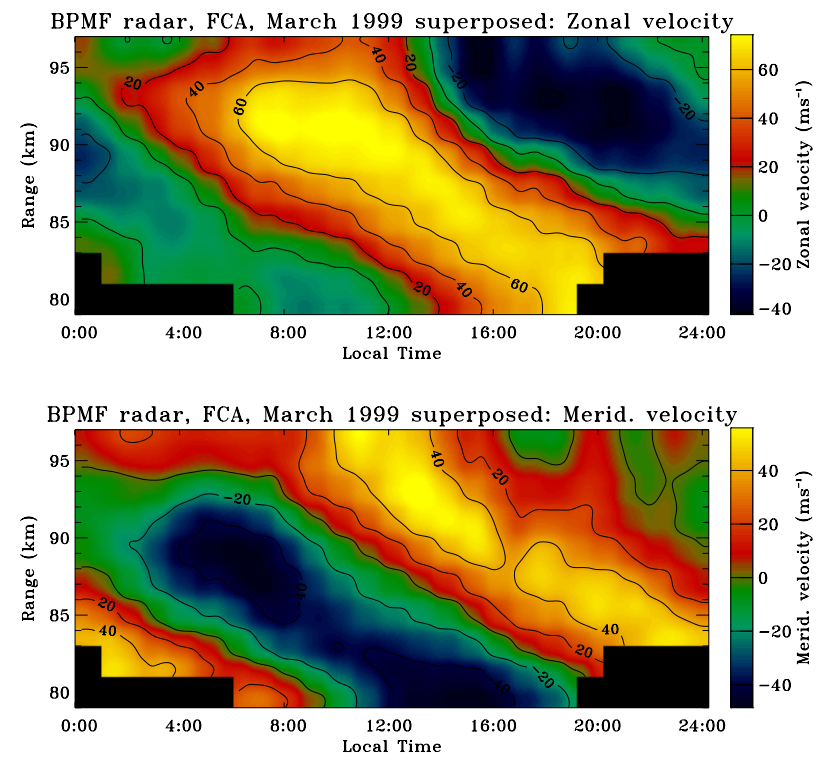

Fig. 5. Superposed epoch plot of the zonal (top) and meridional (bottom) velocity for March 1999.

The harmonic analysis described in Sect. 3.2 has also been applied to half hourly averaged estimates of $v_{\text {fca }}$ throughout 1997. The application of this analysis is intended to determine the phase of maximum $v_{\mathrm{fca}}$ of the 12-, 24- and 48-hour components, and does not assume the components of $v_{\text {fca }}$ will vary sinusoidally. It may be that the square of $v_{\text {fca }}$ (i.e. the turbulent energy dissipation rate) varies sinusoidally. However, the results of Fig. 6 suggest the diurnal component does vary approximately sinusoidally throughout March. As a result, the amplitudes of the fit are used as a quantitative measure of the presence of a particular tidal component, rather than as a measure of the amplitude of the component, and will hereafter be referred to as "apparent" amplitudes. The resulting diurnal tidal apparent amplitudes and phases are shown in Fig. 7. Comparison with the corresponding zonal velocity diurnal tidal phases of Fig. 4 reveals that the agreement between the phases of maximum zonal and turbulent velocities, exhibited in Fig. 6, are observed between January and September at all ranges between $80 \mathrm{~km}$ and $94 \mathrm{~km}$. The apparent amplitudes for the 12- and 48-hour components of $v_{\mathrm{fca}}$ (not shown) are insignificant, suggesting that these components are not present in $v_{\text {fca }}$.

Figure 7 also shows an increase in the amplitude of the diurnal variation of turbulent velocity above $96 \mathrm{~km}$, between September and March, without any accompanying increase in the zonal or meridional diurnal amplitudes. The corresponding phases of maximum amplitudes are around local midnight. These results are most probably due to leakage from E-region total reflection (e.g. Hocking, 1988), producing specular scatter and small turbulent velocities. The E-region height minimises around local midday, suggesting harmonic analysis will result in a diurnal variation of turbulent velocity with a minimum amplitude at midday, and hence, a maximum amplitude at midnight.

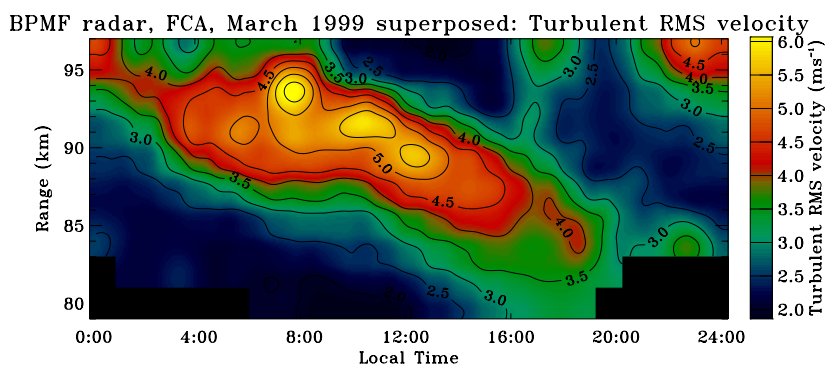

Fig. 6. Superposed epoch plot of the turbulent velocity for March 1999.

\subsection{Descending power layers}

Another phenomenon observed during daytime at around the time of maximum diurnal tide is descending power layers, as illustrated in Fig. 8. These layers have been consistently observed in Adelaide since the installation of the Buckland Park MF radar in the late 1960's (R. A. Vincent, private communication), and are observed by MF radars at other midlatitude sites, such as Christchurch $\left(44^{\circ}\right)$ (e.g. Brown and Fraser, 1996). The improved BPMF radar now allows a study of such layers without compromising the routine FAC analysis described in Sect. 3. The routine analysis as of 1999 incorporates an extra analysis to calculate the power profiles for virtual heights between 60 and $160 \mathrm{~km}$. These results will be described in a subsequent paper.

Figures 8 shows an image plot of power with the FCA true velocity estimates overlaid. Although these results suggest the layer formation at each height commences when the zonal velocity becomes zero, there are a number of days where there appears to be no distinct correlation between the layer formation and the wind components. The reduced number of velocity estimates after the passage of the layer is due to large turbulent velocities and extremely small pattern scales, resulting in low cross-correlations between antennas such that the FCA is unable to provide a velocity estimate.

\section{Discussion}

The results of Fig. 2 suggest that wide-beam MF radar systems produce comparable horizontal velocity estimates and larger $v_{\text {fca }}$ estimates than narrow-beam MF radars. While there is no obvious reason why the horizontal velocity estimated using different beamwidths should differ, this is still a reassuring result. Although the larger turbulent velocities estimated do not necessarily imply that wide-beam width radars overestimate the turbulent velocity, Sect. 2.3 suggests that there are a number of potential causes of overestimation for wide-beam systems. In the absence of any potential reasons why narrow-beam systems should underestimate the turbulent velocity, we conclude that wide-beam systems do, in fact, overestimate the turbulent velocity. Although the sparcity of available results prohibits an estimate of the relative contribution of turbulent velocity and alternative contri- 

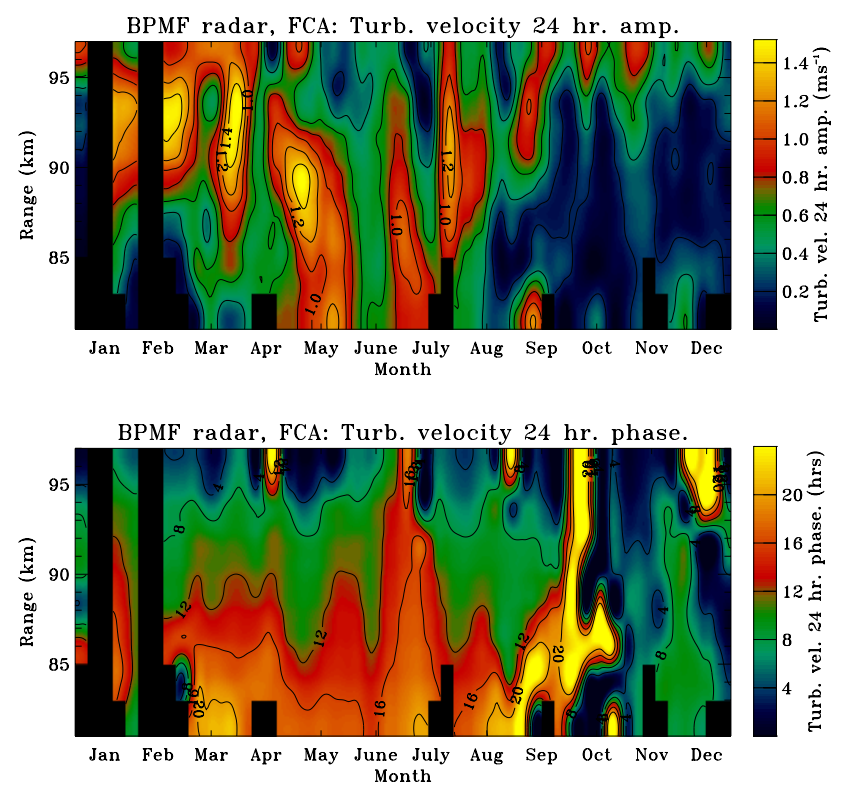

Fig. 7. Magnitude (top) and phase (bottom) of the diurnal variation of the turbulent velocity deduced by harmonic fitting.

butions to the $v_{\mathrm{fca}}$ estimate, a rough subjective interpolation of the results obtained from Fig. 2 suggest that turbulent velocity contribution to the $v_{\text {fca }}$ estimate is approximately $70 \%$ for a $5^{\circ}$ beamwidth. This is comparable to the estimates obtained by Hocking (1988) using the spectral width technique with a comparable beamwidth $\left(4.5^{\circ}\right)$.

A number of authors have previously reported correlations between turbulent velocities (and/or energy dissipation rates) and diurnal tidal amplitudes or wind velocity. Roper (1966) found correlations between diurnal tidal amplitudes and averaged energy dissipation rates, using meteor observations at Adelaide between 80 and $100 \mathrm{~km}$ during 1961. However, no such correlation was observed over the preceding two years. Lesicar (1993) found correlations between wind speed and turbulent velocities in short term FCA results between 80 and $100 \mathrm{~km}$ from Blanchetown $\left(35^{\circ}, 139^{\circ}\right)$ and Christmas Island. The fact that the BPMF to wide-beam turbulent velocity ratios exhibit a larger departure from unity $(0.84)$ than the velocity ratios (1.01 and 1.05) may indicate the FCA turbulent velocity estimates are independent of the horizontal wind velocity estimates. This is an important point in justifying that correlations observed between FCA horizontal wind components (or wind speeds) and turbulent velocities, such as those shown in Fig. 3 and those observed by Lesicar (1993), are real, rather than FCA analysis artifacts.

A number of results similar those shown in Fig. 6 have been previously observed at a number of different sites. During summer observations with the Scott Base MF radar $\left(78^{\circ}\right.$ S, $167^{\circ}$ E) in Antarctica, Fraser and Khan (1990) observed minima in $T_{0.5}$ (i.e. maxima in $v_{\text {fca }}$ ) during the maximum westward extent of the semidiurnal tide. This result corresponds with the PMSE observations of Czechowsky (1989) made using the mobile Sousy radar in Andenes $\left(69^{\circ} \mathrm{N}, 16^{\circ}\right.$

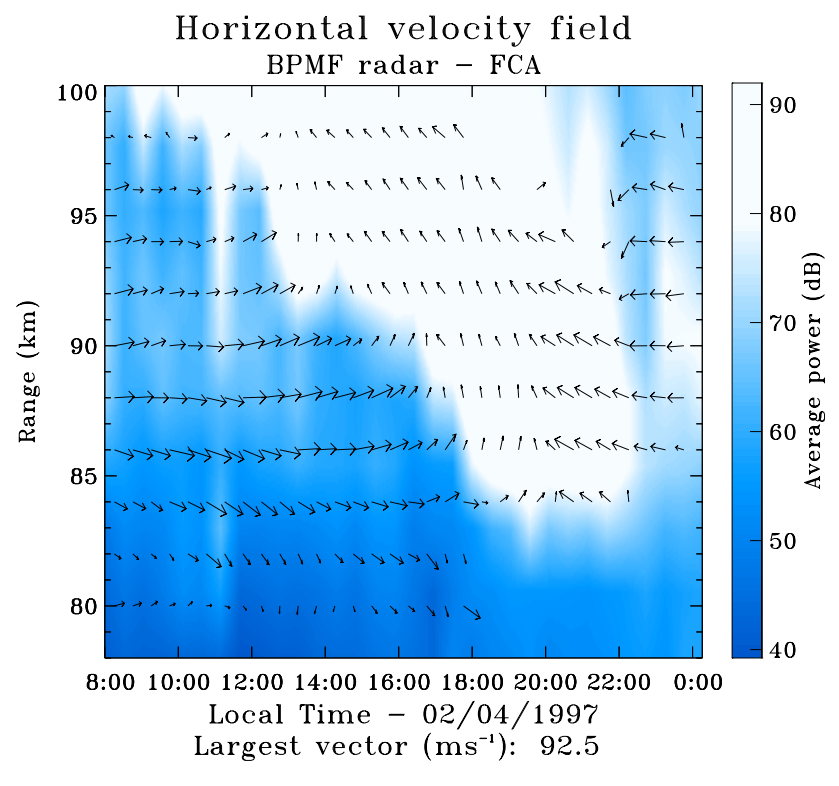

Fig. 8. Power image plot with FCA velocities overlaid for 2nd April 1997.

E), Norway, where an increase in echo power was observed at the time of maximum westward velocity of the semidiurnal tide. However, these results could not be confirmed statistically using all PMSE observations. Fraser and Khan (1990) consider a number of possible explanations for their results. Observations of gravity wave saturation suggest that maximum turbulence due to convective instability has been observed to occur where the temperature gradient was most negative (e.g. Fritts et al., 1988), corresponding to the maximum westward velocity for a westward propagating tide, as observed at Scott Base. Alternatively, tidal modulation of the gravity wave/mean-flow interaction has been proposed.

Figures 5 and 6 show that the turbulent velocity enhancements have a strong diurnal component that is in-phase with the zonal wind component. Tides can destabilize the atmosphere either through modification of the temperature structure and by wind shears and/or by modulating gravity wave breaking (e.g. Liu et al., 2000). The least statically stable regions occur where the temperature height gradient $\mathrm{d} T / \mathrm{d} z=$ $\mathrm{d} T_{0} / \mathrm{d} z+\mathrm{d} T^{\prime} / \mathrm{d} z$, where $T_{0}$ is the ambient temperature and $T^{\prime}$ is the wave induced perturbation, is most negative, which occurs at the phase of the wave where the vertical perturbation velocity $w^{\prime}$ is largest. We note that the vertical descent of $1 \mathrm{~km} \mathrm{~h}^{-1}$ of the wave phase is consistent with the $(1,1)$ mode. Although this mode is westward propagating, and hence, has a net upward flux of westward momentum, at latitudes greater than about $25^{\circ}$ the momentum flux is positive (e.g. Teitelbaum and Vial, 1981). Consequently, at Adelaide, the $u^{\prime}$ and $w^{\prime}$ velocity components will be in-phase, so the maximum negative temperature gradients will occur where the eastward velocity maximizes.

Values of $\mathrm{d} T^{\prime} / \mathrm{d} z$ can be estimated using peak values of $u^{\prime} \approx 60 \mathrm{~m} \mathrm{~s}^{-1}$ (Fig. 5) to scale temperature perturbations 
derived from the GSWM-98 tidal model (e.g. Hagan et al., 1999). Peak values of $T^{\prime} \sim 15^{\circ} \mathrm{K}$ are estimated, producing a maximum negative gradient of $-2 \pi T^{\prime} / \lambda_{z} \approx$ $-4^{\circ} \mathrm{K} \mathrm{km}^{-1}$. This is superimposed on an ambient gradient of about $-1^{\circ} \mathrm{K} \mathrm{km}^{-1}$ during the Fall equinox at similar latitudes to Adelaide (e.g. States and Gardner, 2000; Chen et al., 2000). Although the combined tidal and ambient temperature gradient of $\approx-5^{\circ} \mathrm{K} \mathrm{km}^{-1}$ is insufficient to cause static instability in the mesopause region, it should be noted that the region of maximum $u^{\prime}$ also coincides with the maximum shear in $v^{\prime}$, so it is also a region of dynamic instability. In summary, the least stable, and hence, most turbulent region of the MLT, coincides with the phase of the $u^{\prime}$ component, as observed.

An alternative explanation is that the diurnal variation of the turbulent velocity is due to the change in scattering characteristics produced by descending layers, such as that illustrated in Fig. 8, where it appears that the layer begins forming at the phase of zero zonal velocity, a condition under which sequential sporadic-E is proposed to be formed (e.g. Mathews, 1998). However, when layers such as those shown Fig. 8 are observed, there is no evidence of any corresponding layer at the phase of maximum negative meridional velocity observed during nighttime hours, which is normally observed for sporadic-E. However, nighttime observations are made at a lower PRF $(20 \mathrm{~Hz})$ than daytime $(100 \mathrm{~Hz})$, and the results are consequently noisier, which may mask the detection of nighttime layers. Furthermore, the layer observed in Fig. 8 descends to a height of approximately $84 \mathrm{~km}$, which is considerably lower than the "dumping heights" of approximately $90 \mathrm{~km}$ usually observed for sporadic-E.

The reduced number of velocity estimates observed in Fig. 8 after the passage of the layer is due to large turbulent velocities. There is, therefore, a rapid transition from specular to turbulent scatter. This behavior was also observed by Brown and Fraser (1996), who found periods of low pattern lifetimes (and hence, large turbulent velocities) following the passage of a descending layer detected using frequency domain interferometry (FDI). They propose that this enhanced turbulence may be a result of the breaking of tidal or gravity waves. The current results suggest that the mechanism for generation of enhanced turbulent velocities may differ above and below $94 \mathrm{~km}$. The large turbulent velocities observed after the passage of the layer are observed only above $94 \mathrm{~km}$. This explains why the phase of maximum turbulent velocity seen in Fig. 6, is earlier than the phase of maximum zonal velocity above $94 \mathrm{~km}$, where enhanced turbulence appears to be generated by diurnal tidal breaking before the zonal velocity reaches its eastward maximum. Below $94 \mathrm{~km}$, enhanced turbulence is observed when the zonal velocity reaches its eastward maximum.

\section{Summary and future work}

The use of the full correlation analysis for turbulent velocity estimation has been investigated. Observations made using different beamwidth have confirmed the suggestions of previous authors that wide transmit beam widths lead to an overestimation of the turbulent velocity. The annual variation of the turbulent velocity reveals an increase in turbulent velocity with height, and equinoctal minima and solstice maxima observed below $80 \mathrm{~km}$. Investigations of the turbulent velocities about the March diurnal tide has revealed a diurnal variation in-phase with the zonal velocity which exists between February and September. Descending power layers are also observed during this period.

Future work will include using the "radar backscatter model" of Holdsworth and Reid (1995) to investigate the effects of various radar and atmospheric parameters on the estimated turbulent velocity. The radar backscatter model allows the simulation of the horizontal and vertical turbulent velocities, either together or separately, thereby allowing the investigation of the effects of the horizontal and vertical turbulent velocities to be evaluated separately.

Acknowledgements. The Buckland Park MF radar was supported by Australian Research Council grants A69031462 and A69231890.

Topical Editor D. Murtagh thanks P. Hoffmann and C. Hall for their help in evaluating this paper.

\section{References}

Bracewell, R., The Fourier Transform and its Applications, (McGraw-Hill, New York), 1965.

Briggs, B. H., Radar observations of atmospheric winds and turbulence: A comparison of techniques, J. Atmos. Terr. Phys., 42, 823-833, 1980.

Briggs, B. H., The analysis of spaced sensor records by correlation techniques, in Handbook for MAP, vol. 13, pp. 166-186, SCOSTEP Secr., Univ. of Ill., Urbana, 1984.

Briggs, B. H., Phillips, G. J., and Shinn, D. H., The analysis of observations on spaced receivers of the fading of radio signals, Proc. Phys. Soc., 63B, 106-121, 1950.

Briggs, B. H., Elford, W. G., Felgate, D. G., Golley, M. G., Rossiter, D. E., and Smith, J. W., Buckland Park aerial array, Nature, 223(5213), 1321-1325, 1969.

Brown, W. O. J. and Fraser, G. J., Frequency domain interferometry on spaced antenna MF radar, Radio Sci., 31(5), 1077-1088, 1996.

Chau, J. L., Doviak, R. J., Muschinski, A., and Holloway, C. L., Tropospheric measurements of turbulence and characteristics of Bragg scatterers using the Jicamarca VHF radar, Radio Science, 35(1), 179-193, 2000.

Chen, S., Hu, Z., White, M. A., Chen, H., Krueger, D. A., and She, C. Y., Lidar observations of seasonal variation of diurnal mean temperature in the mesopause region over Fort Collins, Colorado $\left(41^{\circ} \mathrm{N}, 105^{\circ} \mathrm{W}\right)$ J. Geophys. Res., 105, 12371-12379, 2000.

Czechowsky, P., Reid, I. M., Rüster, R., and Schmidt, G., VHF radar echoes observed in summer and winter polar mesosphere over Andoya, J. Geophys. Res., 94, 5199-5217, 1989.

Doviak, R. J., Lataitas, R. J., Holloway, C. L., and Van Baelen, J., A generalized theoretical analysis of cross-correlation and cross-spectra for spaced-antenna wind profilers, NCAR Technical note NCAR/TN-407+STR, National Center for Atmospheric Research, Boulder, Colorado, 1995. 
Fraser, G. J. and Khan, U., Semidiurnal variations in the time scale of irregularities near the Antarctic summer mesopause, Radio Science, 25(5), 997-1003, 1990.

Fritts, D. C., Smith, S. A., Balsley, B. B., and Philbrick, C. R., Example of gravity wave saturation and local turbulence production in the summer mesosphere and lower thermosphere during the STATE experiment, J. Geophys. Res., 93, 7015-7025, 1988.

Hagan, M. E., Burrage, M. D., Forbes, J. M., Hackney, J., Randel, W. J., and Zhang, X., GSWM-98: Results for migrating solar tides, J. Geophys. Res., 104, 6813-6828, 1999.

Hall, C. M., Meek, C. E., and Manson, A. H., Turbulent energy dissipation rates from the University of Tromso/University of Saskatchewan MF radar, J. Atmos. Terr. Phys., 60(4), 437-440, 1998.

Hocking, W. K., On the extraction of atmospheric turbulence parameters from radar backscatter Doppler spectra, I, Theory, J. Atmos. Terr. Phys., 45, 89-102, 1983.

Hocking, W. K., Two years of continuous measurements of turbulence parameters in the upper mesosphere and lower thermosphere made with a 2-MHz radar, J. Geophys. Res., 91(D3), 2475-2491, 1988.

Hocking, W. K., An assessment of the capabilities and limitations or radars in measurements of upper atmosphere turbulence, Adv. Space Res, 17(11), 1137-1147, 1996.

Hocking, W. K., Recent advances in radar instrumentation and techniques for studies of the mesosphere, stratosphere, and troposphere, Radio Science, 32(6), 2241-2270, 1997.

Holdsworth, D. A., Signal analysis with applications to atmospheric radars, Ph.D. thesis, Univ. of Adelaide, Adelaide, Aust., 1995.

Holdsworth, D. A., The influence of instrumental effects upon the full correlation analysis, Radio Sci., 34(3), 643-656, 1999.

Holdsworth, D. A. and Reid, I. M., A simple model of atmospheric radar backscatter: Description and application to the full correlation analysis of spaced antenna data, Radio Sci., 30(4), 12631280, 1995.

Holdsworth, D. A. and Reid, I. M., The Buckland Park MF radar: Description of routine analysis, submitted to Radio Sci., 2001.

Holloway, C. L., Doviak, R. J., and Cohn, S. A., Cross correlations of fileds scattered by horizontally anisotropic refractive index irregularities, Radio Sci., 32(5), 1911-1920, 1997.

Lesicar., D., Study of the structure of partial reflection radar scatterers and their application in atmospheric measurements, Ph.D. thesis, Univ. of Adelaide, Adelaide, Aust., 1993.

Liu, H.-L., Hagan, M. E., and Roble, R. G., Local mean state changes due to gravity wave breaking modulated by the diurnal tide, J. Geophys. Res., 105, 12381-12396, 2000.

Lübken, F.-J., Hillert, W., Lehmacher, G., and von Zahn, U., Experiments revealing small impact on turbulence on the energy budget of the mesopshere and lower thermosphere, J. Geophys. Res., 98, 20369-20384, 1993.

Lübken, F.-J., Seasonal variation of turbulent energy dissipation rates at high latitudes as determine by in situ measurements of neutral density fluctuations, J. Geophys. Res., 102, 1344113456, 1997.

Mathews, J., Sporadic E: current views and recent progress, J. Atmos. Solar Terr. Phys., 60(4), 413-435, 1998.

Meek, C. E., Triangle size effect in spaced antenna wind measurements, Radio Sci., 25(4), 641-648, 1990.

Meek, C. E. and Reid, I. M., A simple model for testing the effects of gravity-wave-produced vertical oscillations of scattering irregularities on spaced-antenna, horizontal drift measurements, in Handbook for MAP, vol. 14, pp. 131-133, SCOSTEP Secr.,
Univ. of Ill., Urbana, 1984.

Meek, C. E., Manson, A. H., and Reid, I. M., Observations of mesospheric wind velocities, 2 , Cross-sections of power spectral density for 48-8 hours, 8-1 hours, and 1 hour to 10 min over $60-110$ km for 1981, Radio Sci, 20, 1383-1402, 1985.

Manson, A. H., Meek, C. E., and Gregory, J. B., Gravity waves of short period (5-90 min) in the lower thermosphere at $52^{\circ} \mathrm{N}$ (Saskatoon, Canada); 1978/1979, J. Atmos Terr. Phys, 43(1), 3544, 1981.

Murphy, D. J., Hocking, W. K., and Fritts, D. C., An assessment of the effect of gravity waves on the width of radar Doppler spectra, J. Atmos Terr. Phys, 56(1), 17-29, 1993.

Müller, H. G., Wind shears in the meteor zone, Planet. Space Sci, 16, 61-90, 1968.

Phillips, G. J. and Spencer, M., The effects of anisometric amplitude patterns in the measurement of ionospheric drifts, Proc. Phys. Soc., Ser. B, 68, 481-492, 1955.

Rees, D., Roper, R. G., Lloyd, K., and Low, C. H., Determination of the structure of the atmosphere between 90 and $250 \mathrm{~km}$ by means of contaminant releases at Woomera, May 1968, Phil. Trans. R. Soc. Lond., 271, 631-663, 1972.

Reid, I. M., Vandepeer, B. G. W., Dillon, S. C., and Fuller, B. M., The new Adelaide medium frequency Doppler radar, Radio Sci., 30(4), 1177-1189, 1995.

Roper, R. G., Atmospheric turbulence in the meteor region, J. Geophys. Res., 71(24), 5785-5792, 1966.

Roper, R. G. and Brosnahan, J., Imaging Doppler interferometry and the measurement of turbulence, Radio Sci., 30(4), 11371148, 1997.

States, R. J. and Gardner, C. S., Thermal structure of the mesopause region $(80-105 \mathrm{~km})$ at $40 \mathrm{deg}$ latitude. Part I: Seasonal variations, J. Atmos. Sci., 57, 66-77, 2000.

Sürücü, F., Kudeki, E., and Rastogi, P. K., Systematic errors in spaced antenna wind estimators under inhomogeneous flow conditions, Radio Sci., 30(4), 837-876, 1995.

Tahara, Y., Yamamoto, M., and Fukao, S., Wind estimation errors of the spaced antenna technique studied with simulations and observations: A case study for the MU radar, Radio Sci., 32(4), 1193-1201, 1997.

Teitelbaum, H. and Vial, F., Momentum transfer to the thermosphere by atmospheric tides, J. Geophys. Res., 86, 9693-9697, 1981.

Vandepeer, B. G. W., A new MF Doppler radar for upper atmospheric research, Ph.D. thesis, Univ. of Adelaide, Adelaide, Aust., 1993.

Vandepeer, B. G. W. and Hocking, W. K., A comparison of Doppler and Spaced Antenna Techniques for the measurement of turbulent energy dissipation rates, Geophys. Res. Lett., 20(1), 17-20, 1993.

Vandepeer, B. G. W. and Reid, I. M., Some preliminary results obtained with the new Adelaide MF Doppler radar, Radio Sci., 30(4), 1191-1203, 1995.

Van Zandt, T. E., Green, J. L., Gage, K. S., and Clark, W. L., Vertical profiles of refractvitiy turbulence structure constant: comparison of observations by the Sunset radar with a new theoretical model, Radio Science, 13, 819-829, 1978.

Vincent, R. A. and Lesicar, D., Dynamics of the equatorial mesosphere: First results with a new generation partial reflection radar, Geophys. Res. Lett., 18(5), 825-828, 1991.

Vincent, R. A., Tsuda, T., and Kato, S., A comparative study of mesospheric solar tides observed at Adelaide and Kyoto, J. Geophys. Res, 93(5), 699-708, 1988. 
Vincent, R. A., Kovalam, S., Fritts, D. C., and Isler, J. R., Longterm MF radar observations of solar tides in the low-latitude mesosphere: Interannual variability and comparisons with the GSWM, J. Geophys. Res., 103(8), 8667-8683, 1998.

Vincent, R. A., May, P. T., Hocking, W. K., Elford, W. G., Candy, B. H., and Briggs, B. H., First results with the Adelaide VHF radar: Spaced antenna studies of tropospheric winds, J. Atmos.
Terr. Phys., 49, 353-366, 1987.

Woodman, R. F. and Guillen, A., Radar observations of winds and turbulence in stratosphere and mesosphere, J. Atmos. Sci., 31, 493-505, 1974.

Wu. Y.-F. and Widdel, H.-U., Turbulent energy dissipation and eddy diffusion coefficients dervied from foil cloud measurements, J. Atmos. Terr. Phys., 51, 497-506, 1989. 